\title{
Molecular Gas in Tidal Dwarf Galaxies: On-going Galaxy Formation
}

J. Braine ${ }^{1}$, P.-A. Duc ${ }^{2}$, U. Lisenfeld ${ }^{3}$, E. Brinks ${ }^{4}$, V. Charmandaris ${ }^{5}$, S. Leon ${ }^{3}$

${ }^{1}$ Observatoire de Bordeaux, UMR 5804, B.P. 89, F-33270 Floirac, France

${ }^{2} C E A / D S M / D A P N I A$, Service d'Astrophysique, Saclay, France

${ }^{3}$ IAA, CSIC, Granada, Spain

${ }^{4}$ INAOE, Puebla, Mexico

${ }^{5}$ Cornell University, Astronomy Department, Ithaca, NY, USA

\begin{abstract}
We investigate the process of galaxy formation as can be observed in the only currently forming galaxies - the so-called Tidal Dwarf Galaxies, hereafter TDGs - through observations of the molecular gas detected via its CO (Carbon Monoxide) emission. Molecular gas is a key element in the galaxy formation process, providing the link between a cloud of gas and a bona fide galaxy. We have now detected $\mathrm{CO}$ in 9 TDGs with an overall detection rate of $80 \%$, showing that molecular gas is abundant in TDGs, up to a few $10^{8} M_{\odot}$. The CO emission coincides both spatially and kinematically with the $\mathrm{HI}$ emission, indicating that the molecular gas forms from the atomic hydrogen where the HI column density is high. A possible trend of more evolved TDGs having greater molecular gas masses is observed, in accord with the transformation of $\mathrm{HI}$ into $\mathrm{H}_{2}$. Although uncertainties are still large for individual objects as the geometry is unknown, we find that the "dynamical" masses of TDGs, estimated from the $\mathrm{CO}$ line widths, do not seem to be greater than the "visible" masses ( $\mathrm{HI}+\mathrm{H}_{2}+$ a stellar component), i.e., TDGs require no dark matter. We provide evidence that TDGs are self-gravitating entities, implying that we are witnessing the ensemble of processes in galaxy formation: concentration of large amounts of gas in a bound object, condensation of the gas, which is atomic at this point, to form molecular gas and the subsequent star formation from the dense molecular component.
\end{abstract}

\section{Introduction}

Tidal Dwarf Galaxies (TDGs) are small galaxies which are currently forming from material ejected from the disks of spiral galaxies through collisions. They allow us to observe processes - galaxy formation and evolution - similar to what occurred in the very early universe but in very local objects. As a consequence, they can be studied with a sensitivity and a resolution inconceivable for highredshift sources. Because galactic collisions can be well reproduced through numerical simulations, it is possible to obtain good age estimates for the indi- 
vidual systems (e.g. Duc et al. 2000). The formation of TDGs is not exactly the same as what happened during the major episode of galaxy formation in that the material which TDGs are made from is "recycled", as it was already part of a galaxy. In particular, the presence of metals, both in gas and as dust, facilitates the cooling of the gas and the formation of $\mathrm{H}_{2}$ molecules. Nevertheless, both for TDGs and in the early universe, the galaxy formation process involves clouds of atomic hydrogen (HI) gas gradually condensing through their own gravity, becoming progressively denser, fragmenting, forming molecular gas from the atomic material, and then forming stars. How this occurs in detail at high redshift is unknown and is one reason for studying TDGs.

Perhaps the least well known of these processes is the transformation of atomic into molecular gas because of the difficulty of observing molecular gas in very low-metallicity environments (e.g. Taylor et al. 1998). Because TDGs condense from matter taken from the outer disks of spiral galaxies, the metallicity of the gas they contain is typically only slightly subsolar as opposed to highly subsolar for small dwarf galaxies (Duc et al. 2000). The metallicity dependent CO lines can thus be used as a probe of the molecular gas content as in spiral galaxies. The study of TDGs influences three areas of astronomy: star formation, dark matter, and galaxy formation.

Much of the material described here has been published in Braine et al. (2000, 2001; hereafter Papers I and II.). Readers are referred to these articles for the lengthy details of the sample as well as many figures showing the $\mathrm{CO}$ spectra. Here we focus on the results and the most recent detection.

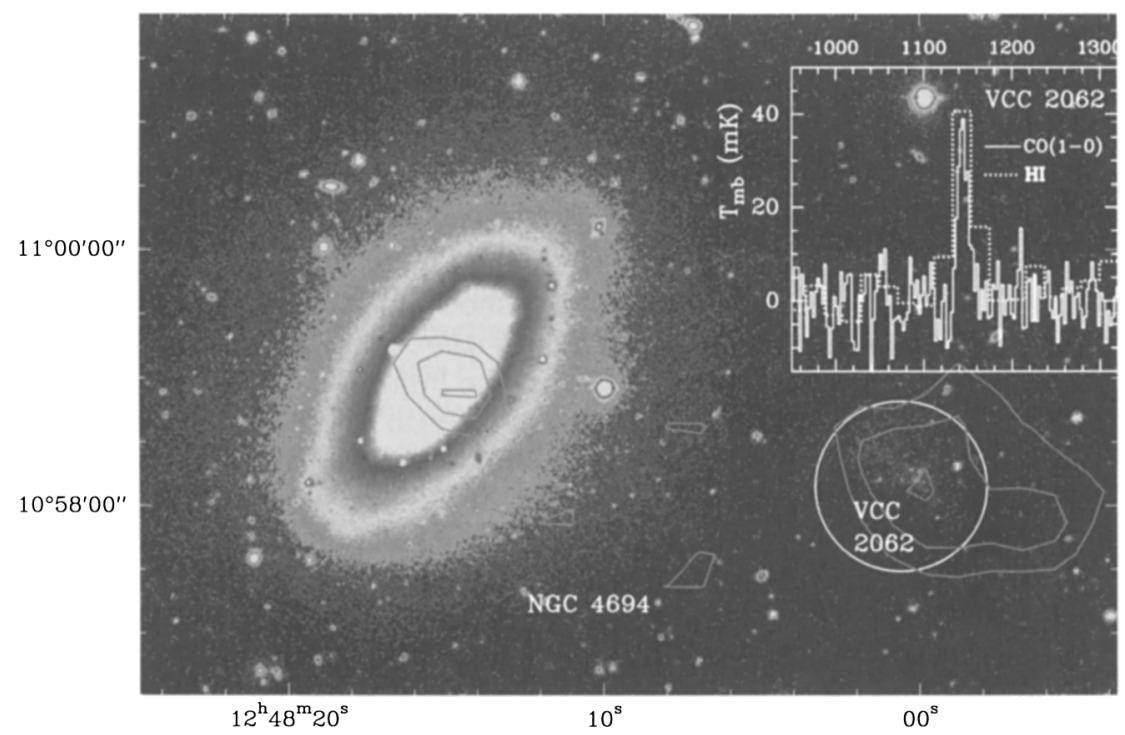

Figure 1. R band image of NGC 4694 and VCC 2062 from Koopman et al. (2001) with the $\mathrm{CO}(1-0)$ and HI spectra of VCC 2062 inset. The angular resolutions are $21^{\prime \prime}$ and $38^{\prime \prime}$ respectively. 


\section{Results}

Our sample consists of interacting systems for which an extensive set of optical and radio (HI) data already exist in the literature. $\mathrm{CO}$ detections were obtained for the following TDGs: VCC 2062 (west of NGC 4694 in the Virgo cluster), NGC 7252 West (hereafter NGC7252W), Arp105S, Arp245N, NGC 4676 North (hereafter NGC4676N), NGC 5291 North and NGC 5291 South (hereafter NGC5291N and NGC5291S), Stephan's Quintet source 'B' (hereafter NGC7319E), and very probably NGC 4038/9 South ("The Antennae", hereafter NGC4038S). Figure 1 shows an R band image of the VCC 2062/NGC 4694 system with contours showing the HI emission from Cayatte et al. (1990). An inset shows the CO and HI spectra at the position of VCC 2062. The other systems are shown in Papers I and II. The western tail of the NGC 2782 (Arp 215) system was also observed with no CO detection, confirming the Smith et al. (1999) non-detection. The western HI tail of NGC 2782 has presumably not had time to condense into $\mathrm{H}_{2}$ and for star formation to begin (see below). We use a $N\left(\mathrm{H}_{2}\right) / I_{\mathrm{CO}}$ factor of $2 \times 10^{20} \mathrm{~cm}^{-2}\left(\mathrm{~K} \mathrm{~km} \mathrm{~s}^{-1}\right)^{-1}$ for all calculations of $\mathrm{H}_{2}$ masses from $\mathrm{CO}$ measurements.

\section{Star Formation in TDGs and other Dwarf Galaxies}

The most striking difference between TDGs and Dwarf Galaxies not identified as tidal is their high CO luminosity (see Fig. 2a), roughly a factor 100 higher than for other dwarf galaxies of similar luminosity and star formation rate (SFR). The most important factor responsible for this difference is certainly the metallicity. As the metallicity increases, the CO lines become optically thick over a larger area and larger velocity range. Furthermore, the shielding against UV radiation due to both CO molecules and dust increases as well. For this reason, a change in metallicity is expected to have a stronger effect in a UV-bright environment (e.g. Wolfire et al. 1993; Braine et al. 1997 Sect 7.3). The details of the comparison dwarf galaxy sample and the SFRs are given in Paper II.

We illustrate some of the differences between TDGs, standard dwarf galaxies, and normal spirals in Fig. 2a, where we show the molecular gas content derived from the $\mathrm{CO}$ luminosity, normalized by the star formation rate (from $\mathrm{H} \alpha$ flux), as a function of luminosity. Whereas the luminosity range of TDGs is indeed typical of dwarf galaxies, their $\mathrm{M}_{\mathrm{mol}} / \mathrm{SFR}$ ratio (equivalent to $\mathrm{CO} / \mathrm{H} \alpha$ ) is rather typical of spirals and much higher (about a factor of 100) than in dwarf galaxies.

It is remarkable that the gas consumption time, the inverse of the SFE, appears not very different in spiral disks, dominated by the stellar mass, and TDGs which are frequently dominated by the gaseous mass and do not share the rotating disk dynamics of spirals.

\section{TDG Formation and the transformation of $\mathrm{HI}$ into $\mathbf{H}_{2}$}

We argue here that it is now possible to follow the TDG formation process from ejection to gravitational collapse to the conversion of $\mathrm{HI}$ into $\mathrm{H}_{2}$ and the subsequent star formation. 

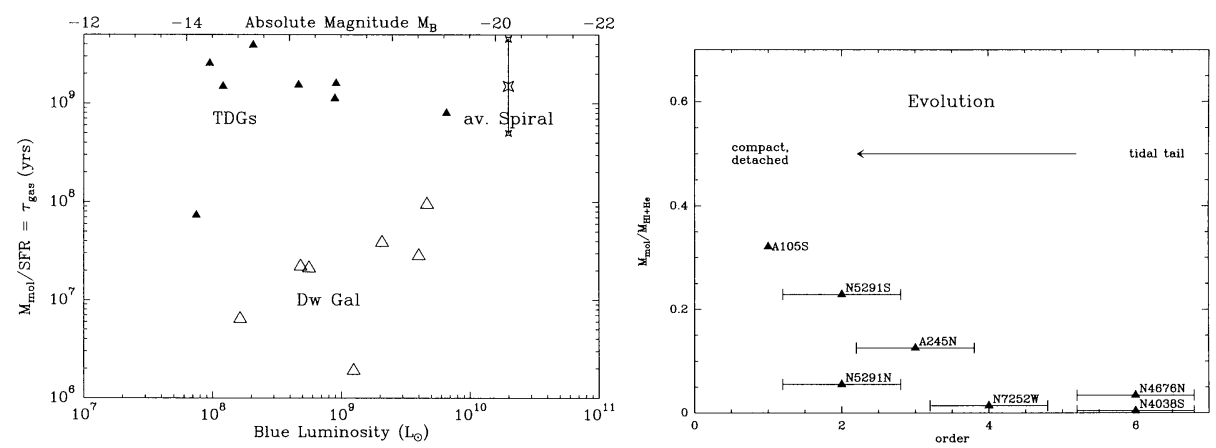

Figure 2. left: Comparison of gas consumption time, $\mathrm{M}_{\mathrm{mol}} / \mathrm{SFR}$ (the inverse of the Star Formation Efficiency (SFE)) as a function of blue luminosity. Small filled triangles represent the TDGs, open triangles the regular dwarf galaxies, and the star an average spiral, similar in position on the diagram to the Milky Way. The smaller stars give the range for "average" spirals. right: Comparison of the $\mathrm{H}_{2} / \mathrm{HI}$ mass ratio with the evolutionary order of the TDGs. NGC7319E was not included due to its unclear morphology. The single non-detection, IC1182E, was also left out. The errorbars indicate the uncertainty in the evolutionary order.

In Paper I, presenting the first $\mathrm{CO}$ detections in the TDGs Arp105S and Arp245N, we ascribed the $\mathrm{CO}$ emission to the formation of molecular gas from the HI. We describe this in more detail below; the calculation of the $\mathrm{H}_{2}$ formation time can be found in Paper II. The time to transform $20 \%$ of the atomic hydrogen into $\mathrm{H}_{2}$ is $t_{20} \approx \frac{10^{7}}{n_{\mathrm{HI}}}$ years. $t_{20}$ is an appropriate indicator because most of the hydrogen gas is still in atomic form, with $20 \%$ being a typical $\mathrm{H}_{2}$ fraction. The $\mathrm{HI}$ will become molecular in the densest parts, staying atomic in less dense regions. The timescale for the $\mathrm{HI} \rightarrow \mathrm{H}_{2}$ conversion is thus much shorter than the other galaxy interaction and formation timescales, of order Myr for typical densities (after some contraction) of $n_{\mathrm{H}} \gtrsim 10 \mathrm{~cm}^{-3}$. The $\mathrm{HI} \rightarrow \mathrm{H}_{2}$ conversion is thus not able to slow down the gravitational collapse.

The average HI column density is probably not very relevant in and of itself. Rather, once the surrounding material is gravitationally contracting, the $\mathrm{HI}$ clouds come closer together and provoke the transformation of $\mathrm{HI}$ into $\mathrm{H}_{2}$, which is what allows star formation to proceed. This is what we see in the TDGs. An interesting counterexample is the western tidal arm (or tail) of NGC 2782. It clearly stems from NGC 2782 and is very HI-rich with many condensations with average column densities of $\mathrm{N}_{\mathrm{HI}} \sim 10^{21} \mathrm{~cm}^{-2}$ over regions several $\mathrm{kpc}$ in size. Neither we nor Smith et al. (1999) detected CO despite the high HI column densities and the presence of disk stars.

NGC 2782 has no single big (TDG-sized) HI condensation at the end of the western tidal tail and indeed the lack of $\mathrm{CO}$ provides a coherent picture: the $\mathrm{HI}$ here is not condensing because the tail is not gravitationally bound and thus $\mathrm{H}_{2}$ is not forming so star formation has not started. In fact, the interaction has certainly added some energy to the tail so we expect that the clouds may 
separate further. In the cases where $\mathrm{CO}$ is detected, the large (TDG) scale is gravitationally bound and even though large amounts of $\mathrm{H}_{2}$ do not form in the outer disks of spirals, $\mathrm{H}_{2}$ forms here because the $\mathrm{HI}$ clouds become closer to each other with time, pushing them to form $\mathrm{H}_{2}$. Along with the almost complete lack of $\mathrm{CO}$ in the outer disks of spirals, the western tail of NGC 2782 is straightforward observational evidence that the $\mathrm{CO}$ is not brought out of spiral disks.

\section{Evolution and morphology}

Assuming TDGs are not short-lived objects, they condense from the tidal tail, the unbound parts of which slowly separate from the TDG. TDGs can then be arranged in a morphological evolutionary sequence which follows $(a)$ their degree of detachment from the tidal tail, which can be roughly defined as the density enhancement with respect to the tidal tail, and $(b)$ the compactness of the object, which is a measure of the degree of condensation of the gas (stars are non-dissipative so the old stellar population, if present, will not "condense"). The classification (evolved, intermediate, young) is simple for a number of objects. Arp105S is clearly very compact and, at the opposite end, NGC4038S and NGC4676N are only just condensing from the tidal tail, being non-compact and with only a small density (light or $\mathrm{HI}$ ) enhancement with respect to the tail. The sequence suggested in Fig. $2 \mathrm{~b}$ represents this evolution.

The conversion of $\mathrm{HI}$ into $\mathrm{H}_{2}$ during contraction suggests that the $\mathrm{H}_{2}$ to $\mathrm{HI}$ mass ratio may also be a tracer of evolutionary state. While a real starburst may blow the gas out of a small galaxy, the $\mathrm{H} \alpha$ luminosities do not suggest that this is the case for the TDGs here. In Fig. $2 \mathrm{~b}$ we plot the molecular-to-atomic gas mass ratio as a function of class, where increasing class indicates less evolved objects. That no known objects occupy the upper right part of the figure is further evidence that the $\mathrm{H}_{2}$ (or $\mathrm{CO}$ ) does not come from pre-existing clouds in spiral disks but rather formed in a contracting object. New high-resolution VLA $\mathrm{HI}$ and Fabry-Perot $\mathrm{H} \alpha$ data should allow subtraction of the tail contribution and enable dynamical, and not purely morphological, criteria to be taken into account (see contributions by Amram and Duc). We will then be able to check and quantify the qualitative evolutionary sequence proposed here.

\section{Dark Matter, CO linewidths and Tidal Dwarf Galaxy masses}

While data are still sparse, we believe there is a correlation between CO line widths and mass indicators. Because $\mathrm{CO}$ is found in the condensed parts of TDGs, it is a better mass indicator than the HI linewidth, for which the contribution of the tidal tails or other unbound material (not TDG) cannot be easily assessed. Clearly, a regular HI (or $\mathrm{CO}$ ) rotation curve would be extremely useful and convincing as a measure of mass.

In order to trace the mass of a system, the material used as a tracer must be $(a)$ gravitationally bound and $(b)$ roughly as extended (or more) as the mass distribution. We believe that CO fulfills these conditions for TDGs. The fact that the $\mathrm{CO}$ is found where the HI column density is high, and that it formed from the condensation of the HI, is good evidence for condition $a$. The second 
condition is more problematic given the large distance and small angular size of most of our sources but nonetheless several considerations lead us to think it is justified. The only extended TDGs, with respect to the resolution of our observations, are NGC4038S and Arp245N. Arp245N is roughly as extended in $\mathrm{CO}$ as at other wavelengths; NGC4038S was only observed at one position. No abundance gradient has been detected or is expected in current TDGs so one may reasonably expect $\mathrm{CO}$ to be visible wherever $\mathrm{HI}$ has condensed into $\mathrm{H}_{2}$. Many dwarf galaxies have very extended HI distributions, up to several times the size of their optical extent. In TDGs, the evidence points to relatively cospatial dense gas and old stellar populations although the relative distributions in the parent disk and collision parameters condition the mass ratio. The diffuse, unbound, $\mathrm{HI}$ in tidal tails is unlikely to form $\mathrm{H}_{2}$ so the molecular component should yield a complete but less confused picture of the dynamics. The old stellar population of TDGs varies greatly but is in general quite dim, furthering the expectation that were DM present in TDGs, we would see it in the gas dynamics.

The apparent, although very rough, correlation between the "Virial masses", $M_{\text {vir }} \approx R \Delta V^{2} / G$, and the $\mathrm{HI}, \mathrm{H}_{2}$, and luminous masses, is shown in Fig. 3 . It is an indication that the line widths are indeed related to mass, analogous to the Tully-Fisher relation for spirals. We infer this principally from the unpopulated lower right (high mass, low linewidth) and upper left (low mass, high linewidth) corners of the panels. In turn, this implies that $(a)$ the objects are kinematically distinct from the parent galaxies and $(b)$ the linewidths can be used as an indicator of mass. We say indicator of mass as opposed to measure of mass because of the great uncertainties, factor 2 or more, in the geometry as well as in the degree of relaxation of the objects. The uncertainty is not symmetric, however, as lines can be widened more easily than narrowed. Low values of $R \Delta V^{2} / G$ are thus significant.

Hibbard \& Barnes (this volume) argue that projection effects can result in inappropriately high virial masses (their expression for $M_{\mathrm{vir}}$ is roughly equivalent to ours) and that we (Braine et al. 2001) have overestimated the contribution of HI to the TDG masses. Their first point is entirely correct and is why our low values of $M_{\text {vir }}$ are significant. Indeed, if projection effects had an effect on CO, then we would find high $M_{\text {vir }}$ values which is not the case and again shows that $\mathrm{CO}$ is an appropriate tracer of the dynamical mass. Inspection of the CO and HI spectra in Braine et al. (2000, 2001), which have similar angular resolutions, shows that only a minority $(0-50 \%$ depending on the object) of the HI (at the $\mathrm{HI}$ peak) is found at velocities outside of the $\mathrm{CO}$ range. One can also see this in Fig. 1 - at the peak, the HI is only slightly wider than the CO such that less than half is outside the $\mathrm{CO}$ velocity range. This cannot change our conclusions. The recent simulations by Bournaud et al. (2003; Duc et al. this volume) confirm again that TDGs condense under their own gravity, which is generated by material from the original spiral disks. Amram et al. (this volume) show that projection effects can be identified kinematically and appear not to be responsible for the appearance of several of the TDGs detected in CO; the TDG candidate in which we did not detect CO, IC $1182 \mathrm{E}$, shows the signs of a projection effect. 


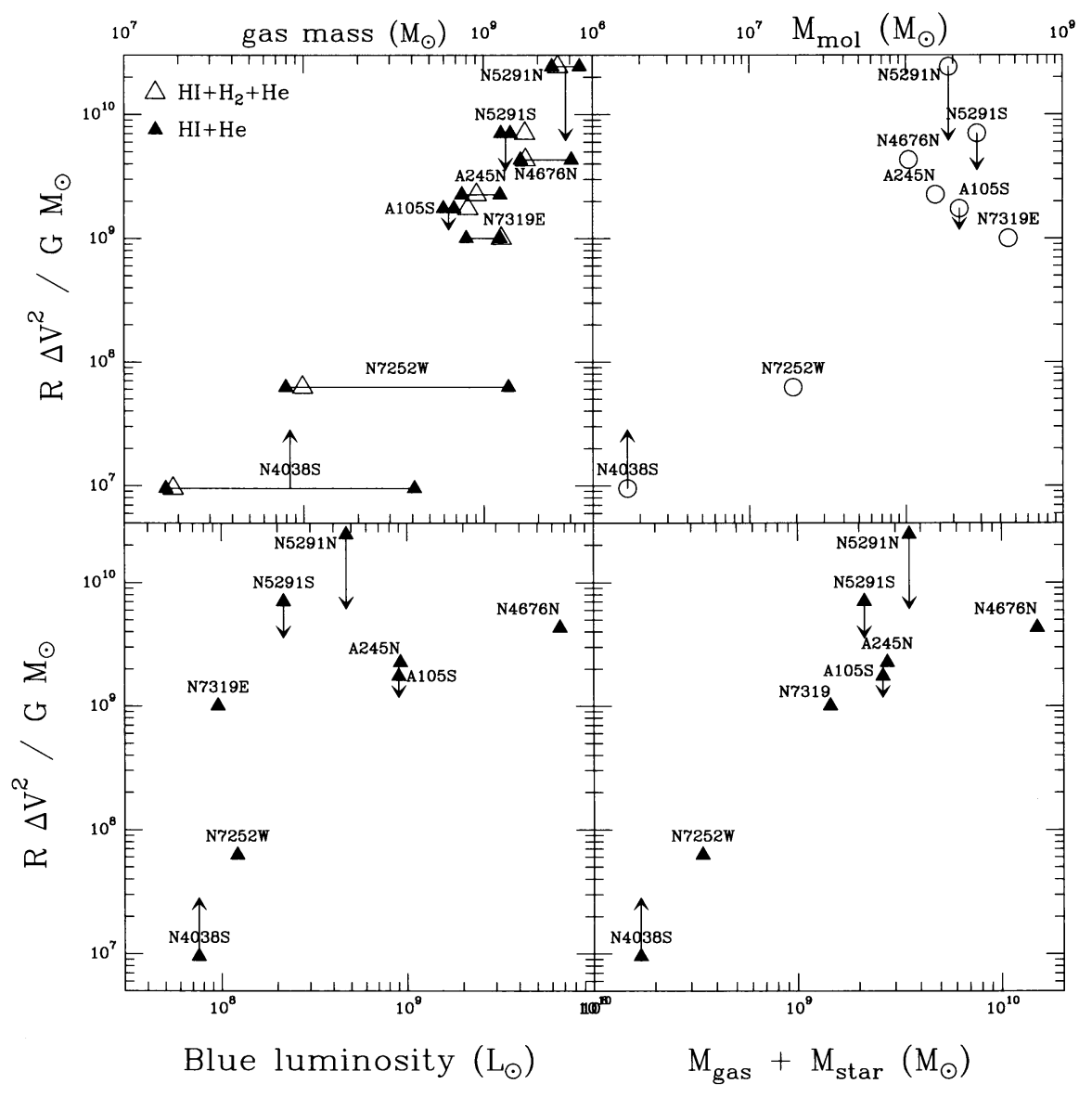

Figure 3. Virial masses derived from CO line widths as a function of $\mathrm{HI}$ and total gas mass (top left), $\mathrm{H}_{2}$ mass (top right), Blue luminosity (lower left), and a combination of all of the above. Ordinate axis expresses the Virial masses as $R \Delta V^{2} / G$. In the first panel the dark triangles joined by a line represent the HI masses within the area observed in $\mathrm{CO}$ and total $\mathrm{HI}$ masses. Open triangles give total gas masses within the $\mathrm{CO}$ beam(s) - these are dominated by the HI. An idea of the uncertainty and whether the value is an overestimate or underestimate is given by the presence of an arrow. A downward arrow is caused by a beam which is large compared to the source size - such that $R_{\mathrm{TDG}}<R_{\text {beam }}$ or that $\Delta V$ is likely an overestimate. NGC4038S is larger than the beam but only one position was measured - hence the up arrow. The position of the end of the arrow is our best estimate, based on source size or line widths in other lines, of where the point should really be placed in the figure. 
Figure 3 shows the variation of the "Virial mass" with total gas mass, molecular gas mass, Blue luminosity, and our best estimate of the total mass. Tidal features are not a homogeneous class - some have virtually no pre-existing stellar component (e.g. NGC5291N) while others (e.g. Arp245N) have a significant contribution from disk stars. To take this into account, we tried to sum the masses of the gaseous and stellar components in the last panel and indeed the trend shows a smaller dispersion. Although the mass to light ratio, $M / L_{B}$, certainly varies within the sample we chose a ratio of $\mathrm{M} / \mathrm{L}_{\mathrm{B}}=2 \mathrm{M} / \mathrm{L}_{\mathrm{B}, \odot}$, midway between that of young and evolved stellar populations.

The "Virial masses" of the sample span a larger range than the gas + star masses. Some of this may be due to the uncertain line width of NGC5291N. Given the uncertainties in line widths and geometry it is too early to make definite statements but so far no dark matter is required to explain the observed $\mathrm{CO}$ line widths. It will be interesting to see whether this remains true when more objects and more precise measurements are available. It is now clear, however, that TDGs can form (condense) under their own gravity and that the uncertainties in our conclusions will decrease steadily as more objects are observed.

If indeed TDGs do not contain DM, then

- they are the only DM-free galaxies identified so far.

- DM is found in the haloes of spiral galaxies.

- TDGs are not representative of the population of Dwarf Galaxies with measured rotation curves as these are quite DM-rich.

\section{References}

Bournaud, F., Duc P.-A. and Masset, F. 2003, A\&A, 411, L469

Braine J., Lisenfeld U., Duc P.-A., Leon S. 2000, Nature, 403, 867

Braine J., Duc P.-A., Lisenfeld U., Charmandaris V., Vallejo O., Leon S., Brinks, E. $2001, A \& A, 378,51$

Braine J., Brouillet N., Baudry A. 1997, A\&A, 318, 19

Cayatte V., van Gorkom J. H., Balkowski C., Kotanyi, C. 1990, AJ, 100, 604

Duc P.-A., Brinks E., Springel V. et al. 2000, AJ, 120, 1238

Koopman R.A., Kenney J.D.P., Young J.S. 2001, ApJS, 135, 125

Smith B. J., Struck C., Kenney J. D. P., Jogee S. 1999, AJ, 117, 1237

Taylor C. L., Kobulnicky H.A., Skillman Evan D. 1998, AJ, 116, 2746

Wolfire M. G., Hollenbach D., Tielens A. G. G. M. 1993, ApJ, 402, 195 